Proceedings of the Estonian Academy of Sciences,

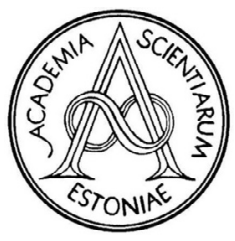

$2021,70,3,235-247$

https://doi.org/10.3176/proc.2021.3.03

CONTROL

Available online at www.eap.ee/proceedings

\title{
Transformation of nonlinear discrete-time state equations into the observer form: extension to non-reversible case
}

\author{
Tanel Mullari ${ }^{\mathrm{a} *}$ and Ülle Kotta ${ }^{\mathrm{b}}$ \\ a Department of Cybernetics, Tallinn University of Technology, Ehitajate tee 5, 19086 Tallinn, Estonia \\ b Department of Software Science, Tallinn University of Technology, Akadeemia tee 21/1, 12618 Tallinn, Estonia
}

Received 4 May 2021, accepted 30 June 2021, available online 15 August 2021

(C) 2021 Authors. This is an Open Access article distributed under the terms and conditions of the Creative Commons AttributionNonCommercial 4.0 International License (http://creativecommons.org/licenses/by-nc/4.0/).

\begin{abstract}
This paper addresses the equivalence under state transformation of a discrete-time nonlinear control system to observer canonical form. Necessary and sufficient conditions for generic equivalence are given for the case when the state equations are not necessarily reversible. The proof is constructive and shows how to find the state transformation if the conditions are satisfied. The derived conditions are then compared with earlier conditions, obtained under more restrictive assumptions, to demonstrate that the earlier result follows directly from our theory. Two examples illustrate the new theory.
\end{abstract}

Key words: nonlinear control system, discrete-time system, observer form, state equivalence, algebraic approach.

\section{INTRODUCTION}

The state transformation of nonlinear state equations into the observer form (state equivalence to observer form) is a recognized approach to state estimation since it allows to construct observers with linear (estimation) error dynamics, converging asymptotically to zero. In spite of this the research area is still active, which is confirmed by numerous recent publications, see [1-6], both for continuous- and discrete-time control systems. The classical observer form is an observable system that is linear up to nonlinear terms depending only on measured inputs and outputs. Most recent papers address the extensions of the classical observer form, since the equivalence conditions for the latter are very restrictive. However, in the discrete-time case there are still a number of unsolved problems regarding the equivalence to the classical observer form. For instance, existing results assume reversibility of state equations with respect to the state variable [7].
In this paper the problem is studied within the new algebraic framework [8] that allows to relax the reversibility assumption. Necessary and sufficient conditions are provided for state equivalence to the classical observer form. The proof is constructive and shows how to construct the necessary state transformation. Moreover, unlike [7], our results are not local but generic, i.e they hold almost everywhere. More precisely, they are valid on an open and dense subset of the state space. Since we look at dimensions (or ranks) over the field of functions, not over $\mathbb{R}$, it is meaningless to speak about constant dimensionality of the distributions. A generic rank is a maximal rank on an open and dense set that may drop on some subset. Reducing the set, one can always achieve a constant rank over $\mathbb{R}$.

Note that a number of results exist for the case when one can easily find the input-output equations via state elimination; see [1] and the references therein. Unfortunately, symbolic state elimination can be sometimes impossible even locally. In a similar manner, the papers [2]

\footnotetext{
*Corresponding author, tanel.mullari@taltech.ee
} 
and [3] do not give state equivalence conditions in terms of the original state equations but characterize it through a certain auxiliary system that is linked to the original system. Unlike [1-3], the goal of this paper was to find the equivalence conditions directly in terms of the original state equations.

\section{PRELIMINARIES}

In this section we recall some preliminary results that originate mostly from [8] and prove some lemmas. Consider the discrete-time single-input single-output (SISO) nonlinear control system

$$
x^{\langle 1\rangle}(t)=\bar{\Phi}(x(t), u(t))
$$

where $x^{\langle 1\rangle}(t):=x(t+1), t \in \mathbb{Z}$, the state variable $x(t) \in$ $\bar{X} \subset \mathbb{R}^{n}$, the control variable $u(t) \in U \subset \mathbb{R}$, and the state transition map $\bar{\Phi}: \bar{X} \times U \rightarrow \bar{X}$ is supposed to be analytic. Both $\bar{X}$ and $U$ are assumed to be open sets. We assume that the map $\bar{\Phi}$ can be extended to the map $\Phi=\left[\bar{\Phi}^{T}, \chi^{T}\right]^{T}: \bar{X} \times U \rightarrow \bar{X} \times \mathbb{R}$ so that $\Phi$ has the global analytic inverse $\left[\Lambda^{T}, \lambda^{T}\right]^{T}: \Phi(\bar{X} \times U) \rightarrow \bar{X} \times U$. Introduce the additional variable at time instant $t, z(t) \in \mathbb{R}$, by

$$
z(t)=\chi(x(t), u(t))
$$

The system (1), (2) defines the inversive difference field $\mathscr{K}$ of meromorphic functions in a finite number of variables from the set $\mathscr{C}=\left\{x, u^{\langle k\rangle}, z^{\langle-l\rangle}, k \geq 0, l \geq 1\right\}$. Here $u^{\langle k\rangle}$ denotes the $k$ th order forward shift of $x$ and $z^{\langle-l\rangle}$ the $l$ th order backward shift of $z$. The 1 st order forward shift of the variable $x$ is defined by equations (1) and the 1 st order backward shifts of $x$ and $u$ by

$$
x^{\langle-1\rangle}=\Lambda\left(x, z^{\langle-1\rangle}\right), \quad u^{\langle-1\rangle}=\lambda\left(x, z^{\langle-1\rangle}\right) .
$$

The forward shift of a function $\varphi\left(x, u, u^{\langle 1\rangle}, \ldots, u^{\langle k\rangle}\right.$, $\left.z^{\langle-1\rangle}, \ldots, z^{\langle-l\rangle}\right) \in \mathscr{K}$ is defined as the composition $\varphi^{\langle 1\rangle}:=\varphi\left(\bar{\Phi}(x, u), u^{\langle 1\rangle}, u^{\langle 2\rangle}, \ldots, u^{\langle k+1\rangle}, \chi(x, u), \ldots, z^{\langle-l+1\rangle}\right)$. The higher order forward shifts of $x$ are defined recursively as

$$
\begin{aligned}
x^{\langle 1\rangle} & =\bar{\Phi}^{1}(x, u):=\bar{\Phi}(x, u), \\
x^{\langle k\rangle} & =\bar{\Phi}^{k}\left(x, u, u^{\langle 1\rangle}, \ldots, u^{\langle k-1\rangle}\right) \\
& :=\bar{\Phi}^{k-1}\left(\Phi(x, u), u^{\langle 1\rangle}, \ldots, u^{\langle k-1\rangle}\right), \quad k \geq 2 .
\end{aligned}
$$

The backward shift of $\varphi$ is the composition $\varphi^{\langle-1\rangle}:=$ $\varphi\left(\Lambda\left(x, z^{\langle-1\rangle}\right), \lambda\left(x, z^{\langle-1\rangle}\right), u, \ldots, u^{\langle k-1\rangle}, z^{\langle-2\rangle}, \ldots, z^{\langle-l-1\rangle}\right)$. The higher order backward shifts of $x$ are defined recursively as

$$
\begin{aligned}
x^{\langle-1\rangle} & =\Lambda^{1}\left(x, z^{\langle-1\rangle}\right):=\Lambda\left(x, z^{\langle-1\rangle}\right) \\
x^{\langle-k\rangle} & =\Lambda^{k}\left(x, z^{\langle-1\rangle}, z^{\langle-2\rangle}, \ldots, z^{\langle-k\rangle}\right) \\
& :=\Lambda^{k-1}\left(\Lambda\left(x, z^{\langle-1\rangle}\right), z^{\langle-2\rangle}, \ldots, z^{\langle-k\rangle}\right), \quad k \geq 2 .
\end{aligned}
$$

Due to (2) and (4), the higher order forward shifts of $z$ can be computed as $z^{\langle k\rangle}=\chi\left(\Phi^{k}\left(x, u, \ldots, u^{\langle k-1\rangle}\right), u^{\langle k\rangle}\right)$, and due to (3) and (5), the higher order backward shifts of $u$ as $u^{\langle-k\rangle}=\lambda\left(\Lambda^{k-1}\left(x, z^{\langle-1\rangle}, \ldots, z^{\langle-k+1\rangle}\right), z^{\langle-k\rangle}\right)$.

Consider the infinite set of symbols $\mathrm{d} \mathscr{C}=$ $\left\{\mathrm{d} x, \mathrm{~d} u^{\langle k\rangle}, \mathrm{d} z^{\langle-l\rangle}, k \geq 0, l \geq 1\right\}$ and let $\mathscr{E}:=\operatorname{span}_{\mathscr{K}}\{\mathrm{d} \mathscr{C}\}$ be the vector space spanned over $\mathscr{K}$ by the elements of $\mathrm{d} \mathscr{C}$, called the 1 -forms

$$
\omega=\sum_{i=1}^{n} A_{i} \mathrm{~d} x_{i}+\sum_{k \geq 0} B_{k} \mathrm{~d} u^{\langle k\rangle}+\sum_{l \geq 1} C_{l} \mathrm{~d} z^{\langle-l\rangle},
$$

where only the finite number of coefficients differs from zero [9]. Define the space $\mathscr{E}^{*}=$ $\operatorname{span}_{\mathscr{K}}\left\{\partial / \partial x, \partial / \partial u^{\langle k\rangle}, k \geq 0, \partial / \partial z^{\langle-l\rangle}, l \geq 1\right\}$, dual to $\mathscr{E}$, whose elements are the vector fields

$$
\Xi=\sum_{i=1}^{n} \xi_{i} \frac{\partial}{\partial x_{i}}+\sum_{k \geq 0} \bar{\xi}_{k} \frac{\partial}{\partial u^{\langle k\rangle}}+\sum_{l \geq 1} \widetilde{\xi}_{l} \frac{\partial}{\partial z^{\langle-l\rangle}} .
$$

By duality between $\mathscr{E}$ and $\mathscr{E} *$ the scalar products of 1forms and vector fields satisfy the following relations:

$$
\left\langle\mathrm{d} x_{i}, \Xi\right\rangle=\xi_{i}, \quad\left\langle\mathrm{~d} u^{\langle k\rangle}, \Xi\right\rangle=\bar{\xi}_{k}, \quad\left\langle\mathrm{~d} z^{\langle-l\rangle}, \Xi\right\rangle=\widetilde{\xi}_{l} .
$$

The backward shift of the vector field $\Xi$ in (6) is the vector field

$$
\Xi^{\langle-1\rangle}=\sum_{i=1}^{n} a_{i} \frac{\partial}{\partial x_{i}}+\sum_{k \geq 0} b_{k} \frac{\partial}{\partial u^{\langle k\rangle}}+\sum_{l \geq 1} c_{l} \frac{\partial}{\partial z^{\langle-l\rangle}},
$$

where

$$
\begin{array}{r}
a_{i}=\left\langle\mathrm{d} x_{i}^{\langle 1\rangle}, \Xi\right\rangle^{\langle-1\rangle}=\left\langle\mathrm{d} \bar{\Phi}_{i}, \Xi\right\rangle^{\langle-1\rangle}, \\
b_{k}=\left\langle\mathrm{d} u^{\langle k+1\rangle}, \Xi\right\rangle^{\langle-1\rangle}, c_{l}=\left\langle\mathrm{d} z^{\langle-l+1\rangle}, \Xi\right\rangle^{\langle-1\rangle} .
\end{array}
$$

The projection of $\Xi$ in (6) is the vector field

$$
\Xi^{\pi}=\sum_{i=1}^{n} \xi_{i} \frac{\partial}{\partial x_{i}}
$$

Note that the shift and projection operators do not commute. A set of vector fields $\Xi_{1}, \ldots, \Xi_{n}$ is called linearly independent if no non-zero coefficients $\beta_{i} \in \mathscr{K}, i=1, \ldots, n$ exist, such that $\sum_{i=1}^{n} \beta_{i} \Xi_{i} \equiv 0$. The vector fields $\Xi_{1}$ and $\Xi_{2}$ commute, if their Lie bracket $\left[\Xi_{1}, \Xi_{2}\right]$ is identically equal to zero. Moreover, the following holds [10]:

$$
\left[\Xi_{1}, \Xi_{2}\right]^{\langle-1\rangle}=\left[\Xi_{1}^{\langle-1\rangle}, \Xi_{2}^{\langle-1\rangle}\right] \equiv 0 .
$$


Lemma 1. If $\Xi \in \operatorname{span}_{\mathscr{K}}\{\partial / \partial x\}$, then for $k=1, \ldots, n$,

$$
\Xi^{\langle-k\rangle} \in \operatorname{span}_{\mathscr{K}}\left\{\frac{\partial}{\partial x}, \frac{\partial}{\partial z^{\langle-1\rangle}}, \ldots, \frac{\partial}{\partial z^{\langle-k\rangle}}\right\} .
$$

Proof. The proof is by induction. Let $k=1$. Compute the first order backward shift of $\Xi=\sum_{i=1}^{n} \xi_{i} \partial / \partial x_{i}$ by (8) and (9). Observe that the only non-zero coefficients in (9) are $a_{i}=\left\langle\mathrm{d} \bar{\Phi}_{i}, \Xi\right\rangle^{\langle-1\rangle}, i=1, \ldots, n, c_{1}=\left\langle\mathrm{d} z^{\langle-1\rangle}, \Xi\right\rangle^{\langle-1\rangle}$, resulting in

$$
\begin{aligned}
\Xi^{\langle-1\rangle}= & \sum_{i=1}^{n} \sum_{j=1}^{n}\left(\frac{\partial \bar{\Phi}_{i}}{\partial x_{j}} \xi_{j}\right)^{\langle-1\rangle} \frac{\partial}{\partial x_{i}} \\
& +\sum_{j=1}^{n}\left(\frac{\partial \chi}{\partial x_{j}} \xi_{j}\right)^{\langle-1\rangle} \frac{\partial}{\partial z^{\langle-1\rangle}} .
\end{aligned}
$$

Suppose next that (12) holds for $k$,

$$
\Xi^{\langle-k\rangle}=\sum_{i=1}^{n} \widehat{\xi}_{i} \frac{\partial}{\partial x_{i}}+\sum_{l=1}^{k} \eta_{l} \frac{\partial}{\partial z^{\langle-l\rangle}} .
$$

The only non-zero coefficients of the backward shift of the above vector field are $a_{i}=\left\langle\mathrm{d} \bar{\Phi}_{i}, \Xi^{\langle-k\rangle}\right\rangle^{\langle-1\rangle}, i=$ $1, \ldots, n, c_{1}=\left\langle\mathrm{d} z, \Xi^{\langle-k\rangle}\right\rangle^{\langle-1\rangle}, c_{l}=\left\langle\mathrm{d} z^{\langle-l+1\rangle}, \Xi^{\langle-k\rangle}\right\rangle^{\langle-1\rangle}=$ $\eta_{l-1}^{\langle-1\rangle}, l=2, \ldots, k+1$, yielding

$$
\begin{aligned}
\Xi^{\langle-k-1\rangle} & =\sum_{i=1}^{n} \sum_{j=1}^{n}\left(\frac{\partial \bar{\Phi}_{i}}{\partial x_{j}} \widehat{\xi}_{j}\right)^{\langle-1\rangle} \frac{\partial}{\partial x_{i}} \\
+ & \sum_{j=1}^{n}\left(\frac{\partial \chi}{\partial x_{j}} \widehat{\xi}_{j}\right)^{\langle-1\rangle} \frac{\partial}{\partial z^{\langle-1\rangle}}+\sum_{l=2}^{k+1} \eta_{l-1}^{\langle-1\rangle} \frac{\partial}{\partial z^{\langle-l\rangle}} .
\end{aligned}
$$

Due to (7) and (10)

$$
\Xi^{\langle-k\rangle}=\Xi^{\langle-k\rangle \pi}+\sum_{q=1}^{k}\left\langle\mathrm{~d} z^{\langle-q\rangle}, \Xi^{\langle-k\rangle}\right\rangle \frac{\partial}{\partial z^{\langle-q\rangle}} .
$$

Lemma 2. If $\Xi \in \operatorname{span}_{\mathscr{K}}\{\partial / \partial x\}$, then $\left(\Xi^{\langle-l\rangle \pi}\right)^{\langle-1\rangle \pi}=$ $\Xi^{\langle-l-1\rangle \pi}$.

Proof. Due to (14), $\quad \Xi^{\langle-l\rangle \pi}=\Xi^{\langle-l\rangle}-$ $\sum_{q=1}^{l}\left\langle\mathrm{~d} z^{\langle-q\rangle}, \Xi^{\langle-l\rangle}\right\rangle \partial / \partial z^{\langle-q\rangle}$. Shifting this equality backward by (8) and (9) gives us $\left(\Xi^{\langle-l\rangle \pi}\right)^{\langle-1\rangle}=$ $\Xi^{\langle-l-1\rangle}-\sum_{q=1}^{l}\left\langle\mathrm{~d} z^{\langle-q\rangle}, \Xi^{\langle-l\rangle}\right\rangle^{\langle-1\rangle} \partial / \partial z^{\langle-q-1\rangle}$. Applying the projection operator to the result proves the lemma.

Finally recall the method to define the new variable $z$ in (2). Compute the Jacobi matrix of $\bar{\Phi}$ in (1) as an $(n \times(n+1))$-matrix of generic rank $n$

$$
T \bar{\Phi}=\left(\frac{\partial \bar{\Phi}}{\partial x} \frac{\partial \bar{\Phi}}{\partial u}\right)
$$

and find its kernel as an 1-dimensional distribution

$$
\operatorname{Ker} T \bar{\Phi}=\operatorname{span}_{\mathscr{K}}\{K\} \subset \operatorname{span}_{\mathscr{K}}\left\{\frac{\partial}{\partial x}, \frac{\partial}{\partial u}\right\}
$$

such that $\left\langle\mathrm{d} \bar{\Phi}_{i}, K\right\rangle \equiv 0$. Then the new variable $z$ in (2) can be computed as the canonical parameter of the vector field $K$ according to

$$
\langle\mathrm{d} \chi, K\rangle \equiv 1
$$

\section{PROBLEM STATEMENT AND THE MAIN RESULT}

Consider the system (1) together with the output function

$$
x^{\langle 1\rangle}=\bar{\Phi}(x, u), \quad y=h(x),
$$

where the output $y(t) \in Y \subseteq \mathbb{R}$ and $Y$ is an open set. The goal is to find, if possible, the state transformation $X=\Psi(x)$, such that the equations (16) in the new coordinates are in the observer form

$$
\begin{aligned}
& X_{i}^{\langle 1\rangle}=X_{i+1}+\varphi_{i}(y, u), \quad i=1, \ldots, n-1, \\
& X_{n}^{\langle 1\rangle}=\varphi_{n}(y, u), \quad y=X_{1} .
\end{aligned}
$$

Denote $y^{\langle 0\rangle}=h(x)$. Compute, using (4),

$$
y^{\langle l\rangle}=h\left(\bar{\Phi}^{l}\left(x, u, \ldots, u^{\langle l-1\rangle}\right)\right), \quad l=1, \ldots, n-1 .
$$

Recall [11] the spaces $\mathscr{Y}:=\operatorname{span}_{\mathscr{K}}\left\{\mathrm{d} y^{\langle l\rangle}, l \geq 0\right\}, \mathscr{U}:=$ $\operatorname{span}_{\mathscr{K}}\left\{\mathrm{d} u^{\langle j\rangle}, j \geq 0\right\}, \mathscr{X}:=\operatorname{span}_{\mathscr{K}}\{\mathrm{d} x\}$. The subspace $\mathscr{O}=\mathscr{X} \cap(\mathscr{Y}+\mathscr{U})$ is called the observable space of system (16).

Assumption 3. The system (16) satisfies the generic observability condition $\operatorname{dim}_{\mathscr{K}} \mathscr{O}=n$.

Define the set of 1-forms:

$$
\omega_{l}:=\sum_{i=1}^{n} \frac{\partial y^{\langle l\rangle}}{\partial x_{i}} \mathrm{~d} x_{i}, \quad l=0, \ldots, n-1 .
$$

Assumption 3 is equivalent to the condition that the 1forms $\omega_{l}, l=0, \ldots, n-1$, are linearly independent:

$$
\operatorname{dim}_{\mathscr{K}}\left(\operatorname{span}_{\mathscr{K}}\left\{\omega_{l}, l=0, \ldots, n-1\right\}\right)=n .
$$

Define the vector field $\Xi \in \operatorname{span}_{\mathscr{K}}\{\partial / \partial x\}$ such that

$$
\left\langle\omega_{l}, \Xi\right\rangle \equiv \delta_{l, n-1}, \quad l=0, \ldots, n-1 .
$$

Since the system (20) defines $\Xi$ in the $n$-dimensional

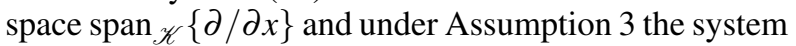
(20) consists of $n$ independent equations, $\Xi$ is uniquely defined. Note that if Assumption 3 is not satisfied, for instance, if the dimension is $n-1$, then $\omega_{n-1}$ is a linear 
combination of $\omega_{l}, l=0, \ldots, n-2$. Then from the equation $\left\langle\omega_{n-1}, \Xi\right\rangle \equiv 1$ follows the contradiction $0=1$ and the system (20) is not solvable.

Recall that by the definition of $\Xi$ and Lemma 1 the vector fields $\Xi^{\langle-k\rangle}, \quad k=0, \ldots, n$, belong to $\operatorname{span}_{\mathscr{K}}\left\{\partial / \partial x, \partial / \partial z^{\langle-1\rangle}, \ldots \partial / \partial z^{\langle-k\rangle}\right\}$. However, one can consider all such vector fields as the elements of a larger dimensional space $\operatorname{span}_{\mathscr{K}}\left\{\partial / \partial x, \partial / \partial z^{\langle-1\rangle}, \ldots . \partial / \partial z^{\langle-n\rangle}\right\}$. In a similar manner one can consider all the 1-forms $\omega_{l}$ and $\mathrm{d} y^{\langle l\rangle}, l=0, \ldots, n-1$, as the elements of the space $\operatorname{span}_{\mathscr{K}}\left\{\mathrm{d} x, \mathrm{~d} u, \ldots, \mathrm{d} u^{\langle n-2\rangle}\right\}$ and write

$$
\begin{aligned}
\mathrm{d} y & =\omega_{0}, \\
\mathrm{~d} y^{\langle l\rangle} & =\omega_{l}+\sum_{j=0}^{l-1} \frac{\partial y^{\langle l\rangle}}{\partial u^{\langle j\rangle}} \mathrm{d} u^{\langle j\rangle}, \quad l=1, \ldots, n-1 .
\end{aligned}
$$

From (14) and (18) follows, for $k=0, \ldots, n, l=0, \ldots, n-1$,

$$
\begin{gathered}
\left\langle\mathrm{d} y^{\langle l\rangle}, \Xi^{\langle-k\rangle}\right\rangle=\left\langle\omega_{l}+\sum_{j=0}^{l-1} \frac{\partial y^{\langle l\rangle}}{\partial u^{\langle j\rangle}} \mathrm{d} u^{\langle j\rangle},\right. \\
\left.\Xi^{\langle-k\rangle \pi}+\sum_{q=1}^{k}\left\langle\mathrm{~d} z^{\langle-q\rangle}, \Xi^{\langle-k\rangle}\right\rangle \frac{\partial}{\partial z^{\langle-q\rangle}}\right\rangle .
\end{gathered}
$$

Take into account that $\Xi^{\langle-k\rangle}$ is the linear combination of $\partial / \partial x_{i}$ and $\omega_{l}$ the linear combination of $\mathrm{d} x_{i}, i=1, \ldots, n$. Then (21) takes, for $k=0, \ldots, n, l=0, \ldots, n-1$, the form

$$
\left\langle\mathrm{d} y^{\langle l\rangle}, \Xi^{\langle-k\rangle}\right\rangle=\left\langle\omega_{l}, \Xi^{\langle-k\rangle \pi}\right\rangle .
$$

Lemma 4. Under Assumption 3 the vector fields $\Xi^{\langle-k\rangle}$, $k=0, \ldots, n-1$, are linearly independent over $\mathscr{K}$.

Proof. To prove the lemma, rewrite (20) according to (22) as

$$
\left\langle\mathrm{d} y^{\langle l\rangle}, \Xi\right\rangle \equiv \delta_{l, n-1}, \quad l=0, \ldots, n-1
$$

The proof is by contradiction. Show first that the vector fields $\Xi$ and $\Xi\langle-1\rangle \pi$ are linearly independent. From (23) we have $\left\langle\mathrm{d} y^{\langle n-2\rangle}, \Xi\right\rangle \equiv 0$. Compare this equality with the formula $\left\langle\mathrm{d} y^{\langle n-2\rangle}, \Xi^{\langle-1\rangle}\right\rangle \equiv 1$, which is obtained via the backward shift of $\left\langle\mathrm{d} y^{\langle n-1\rangle}, \Xi\right\rangle \equiv 1$ and the fact that the backward shift of a scalar product equals the scalar product of the backward shifts of both factors. Due to (14) and taking into account that $y^{\langle n-2\rangle}$ does not depend on $z^{\langle-q\rangle}, q>0$, the last formula can be rewritten as $\left\langle\mathrm{d} y^{\langle n-2\rangle}, \Xi\langle-1\rangle \pi\right\rangle \equiv 1$. Consequently, $\mathrm{d} y^{\langle n-2\rangle}$ annihilates $\Xi$, but not $\Xi^{\langle-1\rangle \pi}$ and so $\Xi$ and $\Xi^{\langle-1\rangle \pi}$ must be linearly independent. The same proof works for $\Xi^{\langle-k\rangle \pi}$, $k=2, \ldots, n-1$.
Lemma 5. [10] Let the vector fields $\Xi_{j}=$ $\sum_{i=1}^{n} \xi_{i j}(x) \partial / \partial x_{i} \in \operatorname{span}_{\mathscr{K}}\{\partial / \partial x\}, j=1, \ldots, n$, be linearly independent over $\mathscr{K}$. If the vector fields $\Xi_{j}$ commute, then, generically, one can define the state transformation $X_{i}=\Psi_{i}(x), \Psi_{i} \in \mathscr{K}, i=1, \ldots, n$, such that

$$
\left\langle\mathrm{d} \Psi_{i}, \Xi_{j}\right\rangle=\delta_{i j}, \quad i, j=1, \ldots, n .
$$

Moreover, in the new coordinates

$$
\Psi_{*} \Xi_{j}=\frac{\partial}{\partial X_{j}}
$$

Note that because the vector fields $\Xi_{j}, j=1, \ldots, n$, span the vector space $\operatorname{span}_{\mathscr{K}}\{\partial / \partial x\}$, the functions $\Psi_{i}(x)$ are uniquely defined.

Theorem 6. (Main result) Under Assumption 3 the system (16) can be transformed via state transformation into the observer form (17) if and only if the following three conditions are satisfied:

(i) the vector fields $\Xi^{\langle-l\rangle \pi}$ commute:

$$
\left[\Xi^{\langle-l\rangle \pi}, \Xi^{\langle-j\rangle \pi}\right] \equiv 0, \quad l, j=0, \ldots, n-1,
$$

(ii) the coefficients of $\Xi^{\langle-l\rangle}, l=0, \ldots, n-1$, depend only on the variable $x$,

(iii) the vector field $\Xi^{\langle-n\rangle \pi}$ is the linear combination of $\Xi^{\langle-l\rangle \pi}, l=0, \ldots, n-1$,

$$
\Xi^{\langle-n\rangle \pi}=\sum_{l=1}^{n} a_{l}\left(y^{\langle-1\rangle}, u^{\langle-1\rangle}\right) \Xi^{\langle-n+l\rangle \pi},
$$

where the coefficients $a_{l}, l=1, \ldots, n$, depend only on the first order backward shifts of $y$ and $u$.

Proof. Sufficiency. Under Assumption 3, $\Xi$ is defined by (20). Then (i), (ii), Lemmas 4 and 5 guarantee the existence of a state transformation $X_{i}=\Psi_{i}(x), i=1, \ldots, n$, such that

$$
\left\langle\mathrm{d} \Psi_{i}, \Xi^{\langle-n+l\rangle \pi}\right\rangle \equiv \delta_{i l}, \quad i, l=1, \ldots, n .
$$

Note that the 1-forms (as the differentials of state transformation) $\mathrm{d} \Psi_{i}, i=1, \ldots, n$, give a basis for $\operatorname{span}_{\mathscr{K}}\{\mathrm{d} x\}$. Moreover, from $(\Psi(x))^{\langle 1\rangle}=\Psi(\bar{\Phi}(x, u))$ one has $\mathrm{d} \Psi\langle 1\rangle \in$ $\operatorname{span}_{\mathscr{K}}\{\mathrm{d} x, \mathrm{~d} u\}$. Therefore, $\mathrm{d} \Psi_{i}^{\langle 1\rangle}, i=1, \ldots, n$, can be expressed as the linear combinations

$$
\mathrm{d} \Psi_{i}^{\langle 1\rangle}(x, u)=\sum_{j=1}^{n} \alpha_{i j}(x, u) \mathrm{d} \Psi_{j}+\beta_{i}(x, u) \mathrm{d} u .
$$

The goal is to prove that if (iii) also holds, then the system (16) has, in the new coordinates, the observer form (17), resulting in

$$
\begin{aligned}
& \mathrm{d} \Psi_{i}^{\langle 1\rangle}=\mathrm{d} \Psi_{i+1}+\mathrm{d} \varphi_{i}(y, u), \quad i=1, \ldots, n-1, \\
& \mathrm{~d} \Psi_{n}^{\langle 1\rangle}=\mathrm{d} \varphi_{n}(y, u) .
\end{aligned}
$$


Recall that $\Psi_{1}=y=h(x)$. Thus, we have to show that under (iii) the only non-zero coefficients $\alpha$ in (26) are

$$
\begin{array}{rlrl}
\alpha_{i 1}(x, u) & =a_{i}(h(x), u), & & i=1, \ldots, n, \\
\alpha_{i, i+1} & \equiv 1, & i=1, \ldots, n-1,
\end{array}
$$

where $a_{i}(y, u)$ are the forward shifts of the coefficients of $\Xi^{\langle-n\rangle}$ in (24). For this purpose compute first, using the right hand side of (26), the scalar products

$$
\begin{aligned}
& \left\langle\mathrm{d} \Psi_{i}^{\langle 1\rangle}, \Xi^{\langle-n+l\rangle \pi}\right\rangle \\
= & \left\langle\sum_{j=1}^{n} \alpha_{i j}(x, u) \mathrm{d} \Psi_{j}+\beta_{i}(x, u) \mathrm{d} u, \Xi^{\langle-n+l\rangle \pi}\right\rangle .
\end{aligned}
$$

From (25) and the fact that $\left\langle\mathrm{d} u, \Xi^{\langle-n+l\rangle}\right\rangle \equiv 0$ we have

$$
\alpha_{i l}(x, u)=\left\langle\mathrm{d} \Psi_{i}^{\langle 1\rangle}, \Xi^{\langle-n+l\rangle \pi}\right\rangle, \quad i, l=1, \ldots, n .
$$

Our next claim is that the coefficients $\alpha_{i l}$ in (29) have the form (28). Prove this first for $l=1$, whereby (29) takes the form

$$
\alpha_{i 1}(x, u)=\left\langle\mathrm{d} \Psi_{i}^{\langle 1\rangle}, \Xi^{\langle-n+1\rangle \pi}\right\rangle, \quad i=1, \ldots, n .
$$

Due to the first formula in (28) we need to show that

$$
\left\langle\mathrm{d} \Psi_{1}^{\langle 1\rangle}, \Xi^{\langle-n+1\rangle \pi}\right\rangle=a_{i}(h(x), u) .
$$

To this end compute the scalar product of $\Xi^{\langle-n\rangle \pi}$ in (24) with $\mathrm{d} \Psi_{1}$, taking into account (25):

$$
\begin{aligned}
\left\langle\mathrm{d} \Psi_{i}, \Xi^{\langle-n\rangle \pi}\right\rangle & =\sum_{l=1}^{n} a_{l}\left(y^{\langle-1\rangle}, u^{\langle-1\rangle}\right)\left\langle\mathrm{d} \Psi_{i}, \Xi^{\langle-n+l\rangle \pi}\right\rangle \\
& =\sum_{l=1}^{n} a_{l}\left(y^{\langle-1\rangle}, u^{\langle-1\rangle}\right) \delta_{i l} .
\end{aligned}
$$

By the definition of the Kronecker delta

$$
\left\langle\mathrm{d} \Psi_{i}, \Xi^{\langle-n\rangle \pi}\right\rangle=a_{i}\left(y^{\langle-1\rangle}, u^{\langle-1\rangle}\right) .
$$

Note that (31) does not follow directly from (32) since the forward shift and the projection operators do not commute $^{1}$. However, by (14), we can write

$$
\Xi^{\langle-n\rangle}=\Xi^{\langle-n\rangle \pi}+\sum_{q=1}^{n}\left\langle\mathrm{~d} z^{\langle-q\rangle}, \Xi^{\langle-n\rangle}\right\rangle \frac{\partial}{\partial z^{\langle-q\rangle}}
$$

and thus, for $i=1, \ldots, n$,

$$
\left\langle\mathrm{d} \Psi_{i}, \Xi^{\langle-n\rangle}\right\rangle=\left\langle\mathrm{d} \Psi_{i}, \Xi^{\langle-n\rangle}\right\rangle,
$$

since $\left\langle\mathrm{d} \Psi_{i}, \partial / \partial z^{\langle-q\rangle}\right\rangle \equiv 0$. By (32), the above results in $\left\langle\mathrm{d} \Psi_{i}, \Xi^{\langle-n\rangle}\right\rangle=a_{i}\left(y^{\langle-1\rangle}, u^{\langle-1\rangle}\right)$ and

$$
\left\langle\mathrm{d} \Psi_{i}^{\langle 1\rangle}, \Xi^{\langle-n+1\rangle}\right\rangle=a_{i}(y, u)
$$

follow easily. Again, from (14) one has

$$
\Xi^{\langle-n+1\rangle}=\Xi^{\langle-n+1\rangle \pi}+\sum_{q=1}^{n-1}\left\langle\mathrm{~d} z^{\langle-q\rangle}, \Xi^{\langle-n+1\rangle}\right\rangle \frac{\partial}{\partial z^{\langle-q\rangle}},
$$

which leads to $\left\langle\mathrm{d} \Psi_{i}^{\langle 1\rangle}, \Xi^{\langle-n+1\rangle}\right\rangle=\left\langle\mathrm{d} \Psi_{i}^{\langle 1\rangle}, \Xi^{\langle-n+1\rangle \pi}\right\rangle=$ $a_{i}(y, u)$. Replacing in the above relation $y$ by $h(x)$ and taking into account (30), we have shown the validity of the first relation in (28).

The second formula in (28) is, by the definition of the Kronecker delta, equivalent to

$$
\alpha_{i l}=\delta_{i, l-1}, \quad i=1, \ldots, n-1, \quad l=2, \ldots, n .
$$

Substituting $\alpha_{i l}$ from (29) into (34), we get, for $i=$ $1, \ldots, n-1, l=2, \ldots, n$,

$$
\left\langle\mathrm{d} \Psi_{i}^{\langle 1\rangle}, \Xi^{\langle-n+l\rangle \pi}\right\rangle \equiv \delta_{i, l-1} .
$$

To prove (35), note that by (25), for $i=1, \ldots, n-1$, $\bar{l}=1, \ldots, n-1$, the following holds:

$$
\left\langle\mathrm{d} \Psi_{i}, \Xi\langle-n+\bar{l}\rangle \pi\right\rangle \equiv \delta_{i \bar{l}}
$$

Even though the shift and projection operators do not commute in a similar manner as above, we can prove that one gets from (36), for $i=1, \ldots, n-1, \bar{l}=1, \ldots, n-1$,

$$
\left\langle\mathrm{d} \Psi_{i}^{\langle 1\rangle}, \Xi\langle-n+\bar{l}+1\rangle \pi\right\rangle \equiv \delta_{i \bar{l}}
$$

Denoting $l:=\bar{l}+1$, we obtain (35), i.e. the validity of the second formula of (28) has been shown. Consequently, (28) holds, and the relation (26) takes the following form:

$$
\begin{aligned}
\mathrm{d} \Psi_{i}^{\langle 1\rangle} & =\mathrm{d} \Psi_{i+1}+a_{i}(h(x), u) \mathrm{d} \Psi_{1}+\beta_{i}(x, u) \mathrm{d} u, \\
i & =1, \ldots, n-1, \\
\mathrm{~d} \Psi_{n}^{\langle 1\rangle} & =a_{n}(h(x), u) \mathrm{d} \Psi_{1}+\beta_{n}(x, u) \mathrm{d} u .
\end{aligned}
$$

As the left hand sides of equalities in (37) are total differentials, the 1 -forms $a_{i}(h(x), u) \mathrm{d} \Psi_{1}+\beta_{i}(x, u) \mathrm{d} u, i=$ $1, \ldots, n$, must be also total differentials. This is possible only if there exist functions $\varphi_{i}\left(X_{1}, u\right), i=1, \ldots, n$, such that

$$
\begin{array}{r}
a_{i}(h(x), u)=\left.\frac{\partial \varphi_{i}\left(X_{1}, u\right)}{\partial X_{1}}\right|_{X_{1}=\Psi_{1}(x)}, \\
\beta_{i}(x, u)=\left.\frac{\partial \varphi_{i}\left(X_{1}, u\right)}{\partial u}\right|_{X_{1}=\Psi_{1}(x)} .
\end{array}
$$

The first relation in (38) is valid if $\Psi_{1}(x)=\sigma(h(x))$.

\footnotetext{
1 Lemma 2 is not applicable either since it addresses the backward shift and not the forward shift.
} 
Next we prove that $\sigma$ is identity function. Recall that by (20) one has $\left\langle\mathrm{d} y^{\langle n-1\rangle}, \Xi\right\rangle \equiv 1$ and shifting this backward $n-1$ times, we get $\left\langle\mathrm{d} y, \Xi^{\langle-n+1\rangle}\right\rangle=\left\langle\mathrm{d} y, \Xi^{\langle-n+1\rangle \pi}\right\rangle \equiv 1$. Due to (25), one has $\left\langle\mathrm{d} \Psi_{1}, \Xi^{\langle-n+1\rangle \pi}\right\rangle \equiv 1$. Therefore, $\sigma=\mathrm{Id}$, resulting in $X_{1}=y=h(x)$.

Form (38) it also follows that the functions $\beta_{i}(x, u)$ depend only on $u$ and $X_{1}=y$, i.e. $\beta_{i}(x, u)=b_{i}(h(x), u)$ and therefore (37) takes the form

$$
\begin{aligned}
\mathrm{d} \Psi_{i}^{\langle 1\rangle} & =\mathrm{d} \Psi_{i+1}+a_{i}(y, u) \mathrm{d} y+b_{i}(y, u) \mathrm{d} u, \\
i & =1, \ldots, n-1, \\
\mathrm{~d} \Psi_{n}^{\langle 1\rangle} & =a_{n}(y, u) \mathrm{d} y+b_{n}(y, u) \mathrm{d} u .
\end{aligned}
$$

Substituting (38) into (39) proves (27).

Necessity. Compute the forward shifts from (17):

$$
\begin{aligned}
& y^{\langle 1\rangle}=X_{2}+\varphi_{1}\left(X_{1}, u\right), \\
& y^{\langle 2\rangle}=X_{3}+\varphi_{2}\left(X_{1}, u\right)+\varphi_{1}\left(X_{2}+\varphi_{1}\left(X_{1}, u\right), u^{\langle 1\rangle}\right),
\end{aligned}
$$

resulting in

$$
\begin{aligned}
& \omega_{0}=\mathrm{d} X_{1}, \\
& \begin{aligned}
\omega_{1}=\mathrm{d} X_{2} & +\frac{\partial \varphi_{1}\left(X_{1}, u\right)}{\partial X_{1}} \mathrm{~d} X_{1}, \\
\omega_{2}=\mathrm{d} X_{3} & +\frac{\partial \varphi_{2}\left(X_{1}, u\right)}{\partial X_{1}} \mathrm{~d} X_{1} \\
& +\left.\frac{\partial \varphi_{1}\left(X_{1}^{\langle 1\rangle}, u^{\langle 1\rangle}\right)}{\partial X_{1}^{\langle 1\rangle}}\right|_{X_{1}^{\langle 1\rangle}=X_{2}+\varphi_{1}\left(X_{1}, u\right)} \\
& \times\left(\mathrm{d} X_{2}+\frac{\partial \varphi_{1}\left(X_{1}, u\right)}{\partial X_{1}} \mathrm{~d} X_{1}\right)
\end{aligned}
\end{aligned}
$$

It means that the 1 -forms $\omega_{k}, k=1, \ldots, n-1$, have the form

$$
\omega_{k}=\mathrm{d} X_{k+1}+\sum_{i=1}^{k} \gamma_{k, i} \mathrm{~d} X_{i} .
$$

Prove first that in the new coordinates $X_{i}, i=1, \ldots, n$, the vector field $\Xi$, defined by (20), has the form

$$
\Xi=\frac{\partial}{\partial X_{n}},
$$

or alternatively,

$$
\left\langle\mathrm{d} X_{i}, \Xi\right\rangle \equiv 0, \quad i=1, \ldots, n-1, \quad\left\langle\mathrm{~d} X_{n}, \Xi\right\rangle \equiv 1 .
$$

The proof is by induction. Observe that $\left\langle\mathrm{d} X_{1}, \Xi\right\rangle \equiv 0$ since $\mathrm{d} X_{1}=\omega_{0}$. Assume next that

$$
\left\langle\mathrm{d} X_{i}, \Xi\right\rangle \equiv 0, \quad i=1, \ldots, k \leq n-2,
$$

and show that

$$
\left\langle\mathrm{d} X_{k+1}, \Xi\right\rangle \equiv 0 \text {. }
$$

Due to (40), $\left\langle\omega_{k}, \Xi\right\rangle=\left\langle\mathrm{d} X_{k+1}, \Xi\right\rangle+\sum_{i=1}^{k} \gamma_{k, i}\left\langle\mathrm{~d} X_{i}, \Xi\right\rangle$. The left hand side of this equality is identically equal to zero due to (20) and the second term on the right hand side is identically equal to zero by (43). Therefore (44) holds. To prove that $\left\langle\mathrm{d} X_{n}, \Xi\right\rangle \equiv 1$, note that $\left\langle\omega_{n-1}, \Xi\right\rangle=\left\langle\mathrm{d} X_{n}, \Xi\right\rangle+\sum_{i=1}^{n-1} \gamma_{n-1, i}\left\langle\mathrm{~d} X_{i}, \Xi\right\rangle$. The left hand side is identically equal to 1 due to (20) and the second term on the right hand side is identically equal to zero due to the first equality of (42). That is, $\left\langle\mathrm{d} X_{n}, \Xi\right\rangle \equiv 1$ and the validity of the second equality of (42) has been proved.

Prove next that

$$
\Xi^{\langle-n+i\rangle \pi}=\frac{\partial}{\partial X_{i}}, \quad i=1, \ldots, n-1 .
$$

Compute, combining (8), (9) and (10),

$$
\left(\frac{\partial}{\partial X_{n}}\right)^{\langle-1\rangle \pi}=\sum_{i=1}^{n}\left\langle\mathrm{~d} X_{i}^{\langle 1\rangle}, \Xi\right\rangle^{\langle-1\rangle} \frac{\partial}{\partial X_{i}} .
$$

According to (17)

$$
\begin{aligned}
& \mathrm{d} X_{i}^{\langle 1\rangle}=\mathrm{d} X_{i+1}+\mathrm{d} \varphi_{i}\left(X_{1}, u\right), \quad i=1, \ldots, n-1, \\
& \mathrm{~d} X_{n}^{\langle 1\rangle}=\mathrm{d} \varphi_{n}\left(X_{1}, u\right) .
\end{aligned}
$$

Substituting (47) into (46), we see that the only non-zero scalar product in (46) is $\left\langle\mathrm{d} X_{n-1}^{\langle 1\rangle}, \partial / \partial X_{n}\right\rangle \equiv 1$, and thus

$$
\Xi^{\langle-1\rangle \pi}=\frac{\partial}{\partial X_{n-1}} .
$$

Computing recursively the backward shifts of $\partial / \partial X_{n-1}$ and so on, we prove (45). Since the partial derivative operators commute, the conditions (i) and (ii) hold.

To prove (iii), observe that by (45), $\Xi^{\langle-n+1\rangle \pi}=$ $\left(\partial / \partial X_{n}\right)^{\langle-n+1\rangle \pi}=\partial / \partial X_{1}$. Shift this vector field backward and compute its projection, taking into account Lemma 2:

$$
\begin{aligned}
\left(\frac{\partial}{\partial X_{n}}\right)^{\langle-n\rangle \pi} & =\left(\frac{\partial}{\partial X_{1}}\right)^{\langle-1\rangle \pi} \\
& =\sum_{i=1}^{n}\left\langle\mathrm{~d} X_{i}^{\langle 1\rangle}, \frac{\partial}{\partial X_{1}}\right\rangle^{\langle-1\rangle} \frac{\partial}{\partial X_{i}}
\end{aligned}
$$

Substituting $\mathrm{d} X_{i}^{\langle 1\rangle}$ from (47) into (48), and taking into account that $X_{1}=y$, results in

$$
\left(\frac{\partial}{\partial X_{n}}\right)^{\langle-n\rangle \pi}=\sum_{i=1}^{n} \frac{\partial \varphi_{i}\left(y^{\langle-1\rangle}, u^{\langle-1\rangle}\right)}{\partial y^{\langle-1\rangle}} \frac{\partial}{\partial X_{i}}
$$


By (41) and (45) above, we get

$$
\Xi^{\langle-n\rangle \pi}=\sum_{i=1}^{n} \frac{\partial \varphi_{i}\left(y^{\langle-1\rangle}, u^{\langle-1\rangle}\right)}{\partial y^{\langle-1\rangle}} \Xi^{\langle-n+i\rangle \pi}
$$

meaning that the condition (iii) also holds.

If the conditions (i) and (ii) of Theorem 6 are satisfied, then the new coordinates $X=\Psi(x)$ can be computed using the method from [10], recalled below. Define an $(n \times n)$-matrix $M$, with columns being the vector fields $\Xi^{\langle-n+i\rangle \pi}, i=1, \ldots, n$ :

$$
M=\left[\begin{array}{lll}
\Xi^{\langle-n+1\rangle \pi} \ldots \Xi^{\langle-1\rangle \pi} \Xi
\end{array}\right]
$$

and compute its inverse $M^{-1}$. Due to (25), the rows of $M^{-1}$ are total differentials of the new state variables $X=\Psi(x)$ :

$$
M^{\langle-1\rangle}=\left[\begin{array}{llll}
\mathrm{d} \Psi_{1}^{T} & \ldots & \mathrm{d} \Psi_{n-1}^{T} & \mathrm{~d} \Psi_{n}^{T}
\end{array}\right]^{T} .
$$

The condition (iii) of Theorem 6 is difficult to check directly if $\Xi^{\langle-n+i\rangle \pi}, i=0, \ldots, n$, have complicated form. In addition to checking the linear independence, the special form of the coefficients $a_{i}$ in (24) has to be shown. Below a formula is given to compute the coefficients $a_{i}$. Rewrite (24) in the matrix form, taking into account (49): $\Xi^{\langle-n\rangle} \pi=M\left[\begin{array}{lll}a_{1} & \ldots & a_{n}\end{array}\right]^{T}$. Under the conditions (i) and (ii) $M^{-1}$ exists and hence

$$
\left[\begin{array}{lll}
a_{1} & \ldots & a_{n}
\end{array}\right]^{T}=M^{-1} \Xi^{\langle-n\rangle \pi} .
$$

\section{THE CHOICE OF $Z$}

In this section we discuss, for an observable system (16), the possible choices of the new variable $z$ in (2). If $\operatorname{rank}_{\mathscr{K}}(\partial \Phi / \partial x)=n$, then the natural choice is $z=u$ [8]. Lemma 8 below shows the possible choice for nonreversible system, i.e. when the choice $z=u$ is impossible. Moreover, we give a corollary from Theorem 6 for reversible systems. The main difference is that in case $z=u$, there is no need to use the projections of the vector fields unlike in the general case. Hence, the vector field $\Xi^{\langle-n\rangle}$ depends only on $\Xi$, resulting in that the condition (iii) in Theorem 6 will be redundant.

As mentioned in Section 2, $T \bar{\Phi}$ is an $[n \times(n+1)]$ matrix with the generic rank $n$, and thus the generic rank of none of its $(n \times n)$-submatrices can be less than $n-1$.

Assumption 7. $\operatorname{rank}_{\mathscr{K}}(\partial \bar{\Phi} / \partial x)=n-1$.

Next consider the non-reversible system that satisfies Assumption 7 .

Lemma 8. If the system (16) is transformable into the observer form (17) and Assumption 7 holds, then one can take $z=y=h(x)$.
Proof. Under the assumption of the lemma, the system (16) takes, in the new coordinates $X=\Psi(x)$, the form (17). Denote the right hand side of the state equations in (17) by $\widetilde{\Phi}(X, u)$ and compute its Jacobi matrix as

$$
T \widetilde{\Phi}=\left(\begin{array}{cccccc}
\frac{\partial \varphi_{1}}{\partial X_{1}} & 1 & 0 & \ldots & 0 & \frac{\partial \varphi_{1}}{\partial u} \\
\vdots & \vdots & \vdots & \vdots & \vdots & \vdots \\
\frac{\partial \varphi_{n-1}}{\partial X_{1}} & 0 & 0 & \ldots & 1 & \frac{\partial \varphi_{n-1}}{\partial u} \\
\frac{\partial \varphi_{n}}{\partial X_{1}} & 0 & 0 & \ldots & 0 & \frac{\partial \varphi_{n}}{\partial u}
\end{array}\right)
$$

In the new coordinates the state equations still satisfy the submersivity assumption, i.e. $\operatorname{rank}_{\mathscr{K}} T \widetilde{\Phi}=n$. Assumption 7 reads in the new coordinates as $\operatorname{rank}_{\mathscr{K}}(\partial \widetilde{\Phi} / \partial X)=$ $n-1$. Due to (51), this is possible only if $\partial \varphi_{n} / \partial X_{1} \equiv$ 0 . The submersivity of the system (17) then yields $\partial \varphi_{n} / \partial u \not \equiv 0$. Find now in coordinates $X$ the basis vector field of $\operatorname{Ker} T \widetilde{\Phi}$, denoted by $K$, from $\langle T \widetilde{\Phi}, K\rangle \equiv 0$. Though $K$ is not uniquely defined, one can take

$$
K=\frac{\partial}{\partial X_{1}}-\left(\frac{\partial \varphi_{n}}{\partial u}\right)^{-1} \sum_{i=2}^{n}\left(\frac{\partial \varphi_{i-1}}{\partial X_{1}} \frac{\partial \varphi_{n}}{\partial u}-\frac{\partial \varphi_{i-1}}{\partial u} \frac{\partial \varphi_{n}}{\partial X_{1}}\right) \frac{\partial}{\partial X_{i}}
$$

Computing the variable $z$ in the new coordinates from (15), i.e. from $\langle\mathrm{d} \chi, K\rangle \equiv 1$, one possible choice is $z=$ $X_{1}=h(x)$.

\subsection{Corollary from Theorem 6: the case of reversible systems}

Assumption 9. The system (1) is generically reversible, i.e.

$$
\operatorname{rank}_{\mathscr{K}}\left(\frac{\partial \bar{\Phi}(x, u)}{\partial x}\right)=n
$$

Under Assumption 9 one can always take $z=u$, though some other options may be possible. In this subsection we will present a corollary from Theorem 6 under Assumption 9 and the choice $z=u$. First, we will give some simple lemmas.

Lemma 10. If $z=u$, then for the vector field $\widehat{\Xi} \in$ $\operatorname{span}_{\mathscr{K}}\{\partial / \partial x\}, \quad \widehat{\Xi}^{\langle-1\rangle}=\widehat{\Xi}^{\langle-1\rangle}$.

Proof. If $z=u$, then the formulae (8) and (9) give

$$
\widehat{\Xi}^{\langle-1\rangle}=\sum_{i=1}^{n}\left\langle\mathrm{~d} \bar{\Phi}_{i}, \widehat{\Xi}\right\rangle^{\langle-1\rangle} \frac{\partial}{\partial x_{i}},
$$

from which the proof directly follows. 
Lemma 11. If $z=u$, then

$$
\begin{aligned}
& \left(\frac{\partial}{\partial u}\right)^{\langle-1\rangle} \\
& =\left.\sum_{i=1}^{n} \frac{\partial \bar{\Phi}_{i}\left(x^{\langle-1\rangle}, u^{\langle-1\rangle}\right)}{\partial u^{\langle-1\rangle}}\right|_{x^{\langle-1\rangle}=\Lambda\left(x, u^{\langle-1\rangle}\right)} \frac{\partial}{\partial x_{i}}+\frac{\partial}{\partial u^{\langle-1\rangle}} .
\end{aligned}
$$

Proof. The proof is the straightforward consequence of the formulae (8) and (9).

Corollary 12. Under Assumption 9, if $z=u$, the conditions of Theorem 6 are equivalent to the conditions:

$\left(i^{*}\right)\left[\Xi, \Xi^{\langle-l\rangle}\right] \equiv 0, \quad l=1, \ldots, n-1$,

(ii $\left.{ }^{*}\right)$ the coefficients of $\Xi^{\langle-l\rangle}, l=0, \ldots, n-1$, depend only on $x$.

Proof. Due to Lemma 10, the equivalence of (ii) and (ii*) is obvious. Moreover, by this lemma we may omit the projections, i.e. the condition (i) takes the form

$$
\left[\Xi^{\langle-l\rangle}, \Xi^{\langle-j\rangle}\right] \equiv 0, \quad l, j=0, \ldots, n-1 .
$$

As (i*) is a direct consequence from (53), we have to prove also the opposite. Actually it is sufficient to prove that from (i*) follows for $\bar{l}=0, \ldots, n-2, \bar{j}=\bar{l}+1, \ldots, n-1$,

$$
\left[\Xi^{\langle-\bar{l}\rangle}, \Xi^{\langle-\bar{j}\rangle}\right] \equiv 0
$$

i.e. for each vector field $\Xi\langle-\vec{l}\rangle$ we do not have to show its commutativity with all $\Xi^{\langle-\hat{j}\rangle}$, but it is sufficient to prove that it commutes with the higher order backward shifts. The reason is that Lie bracket is anti-symmetric and when equal to zero, its value does not depend on the order of vector fields. One can easily get (54) if we rewrite $\left(i^{*}\right)$ as $[\Xi, \Xi\langle-\bar{j}+\bar{l}\rangle] \equiv 0$ for $\bar{j}=\bar{l}+1, \ldots n-1$, $\bar{l}=0, \ldots, n-2$. Though the number of Lie brackets seems to be here larger than in $\left(i^{*}\right)$, it is really not the case, since most of them appear repeatedly. Taking into account (11), apply the $\bar{l}$-th order backward shift to the last equality to get (54).

Now we will show that in case $z=u$, unlike in the general case, the validity of (iii) follows directly from (i) and (ii). This will be performed in three steps. We will show that if (i) and (ii) hold, then

(1) $X_{1}=y$,

(2) $\Xi^{\langle-n\rangle}=a_{n} \Xi, \quad a_{n} \in \mathscr{K}$,

(3) $a_{n}$ is a function of $X_{1}^{\langle-1\rangle}$ and $u^{\langle-1\rangle}$ only.

Note that due to Theorem 6, if (i) and (ii) hold, the state transformation $X=\Psi(x)$ is defined by (25), where, by Lemma 10, we may omit projections. The first step is to show that the above yields $y=X_{1}$, or equivalently,

$$
\left\langle\mathrm{d} y, \Xi^{\langle-l\rangle}\right\rangle \equiv \delta_{l, n-1}, \quad l=0, \ldots, n-1 .
$$

Observe that (56) follows from shifting back (23) $l$ times.
At the second step we will show that (55) is true. Note that Lemmas 4 and 10 give

$$
\operatorname{dim}_{\mathscr{K}}\left(\operatorname{span}_{\mathscr{K}}\left\{\Xi^{\langle-l\rangle}, l=0, \ldots, n-1\right\}\right)=n .
$$

It means that the vector fields $\Xi^{\langle-l\rangle}, l=0, \ldots, n-1$, provide the basis for $\operatorname{span}_{\mathscr{K}}\{\partial / \partial x\}$. By Lemma 10, $\Xi^{\langle-n\rangle} \in \operatorname{span}_{\mathscr{K}}\{\partial / \partial x\}$, and as such, can be expressed as the linear combination of the basis vector fields. Now, shifting (57) backward and denoting $\bar{l}:=l+1$, we obtain

$$
\operatorname{dim}_{\mathscr{K}}\left(\operatorname{span}_{\mathscr{K}}\{\Xi\langle-\bar{l}\rangle, \bar{l}=1, \ldots, n\}\right)=n,
$$

i.e. $\Xi^{\langle-n\rangle}$ and $\Xi^{\langle-\bar{l}\rangle}, \bar{l}=1, \ldots, n-1$, are linearly independent. Therefore, $\Xi^{\langle-n\rangle}$ depends only on $\Xi$, i.e. (55) holds.

At the third step we will prove that $a_{n}$ depends only on $X_{1}^{\langle-1\rangle}$ and $u^{\langle-1\rangle}$. First, we will show that it depends on the variables $x$ and $u^{\langle-1\rangle}$. Express $\Xi^{\langle-n\rangle}$, using (8) and (9), as follows:

$$
\Xi^{\langle-n\rangle}=\sum_{i=1}^{n}\left\langle\mathrm{~d} \bar{\Phi}_{i}, \Xi^{\langle-n+1\rangle}\right\rangle^{\langle-1\rangle} \frac{\partial}{\partial x_{i}} .
$$

Note that the coefficients of $\mathrm{d} \bar{\Phi}_{i}$ depend only on $x$ and $u$, and the coefficients of $\Xi^{\langle-n+1\rangle}$ depend, due to (ii), only on $x$. Consequently, their scalar product depends only on $x$ and $u$ and its backward shift is, according to (3), the function of $x$ and $u^{\langle-1\rangle}$ only. Consequently, the left hand side of (55) depends only on the variables $x$ and $u^{\langle-1\rangle}$. Due to (ii), the coefficients of $\Xi$ depend only on $x$ and therefore, the coefficient $a_{n}$ can not depend on the other variables except $x$ and $u^{\langle-1\rangle}$.

Next, we will show that $a_{n}$ depends only on $X_{1}^{\langle-1\rangle}$ and $u^{\langle-1\rangle}$, or equivalently, that its forward shift $a_{n}^{\langle 1\rangle}(x, u)=$ $a_{n}(\bar{\Phi}(x, u), u)$ depends only on $X_{1}=\Psi_{1}(x)$ and $u$, yielding

$$
\mathrm{d} a_{n}^{\langle 1\rangle}(x, u) \in \operatorname{span}_{\mathscr{K}}\left\{\mathrm{d} \Psi_{1}, \mathrm{~d} u\right\} .
$$

Due to (25) and Lemma 10, in case $z=u$, one has $\left\langle\mathrm{d} \Psi_{1}, \Xi^{\langle-l\rangle}\right\rangle \equiv 0$ for $l=0, \ldots, n-2$, and obviously also $\left\langle\mathrm{d} u, \Xi^{\langle-l\rangle}\right\rangle \equiv 0$ holds. Therefore, (58) is equivalent to

$$
\left\langle\mathrm{d} a_{n}^{\langle 1\rangle}, \Xi^{\langle-l\rangle}\right\rangle \equiv 0, \quad l=0, \ldots, n-2 .
$$

In order to prove the validity of (59), note that from (53) follows

$$
\left[\Xi^{\langle-l\rangle}, \Xi^{\langle-n+1\rangle}\right] \equiv 0, \quad l=0, \ldots, n-2 .
$$

Shifting (60) backward according to (11) and denoting $\bar{l}:=l+1$, we obtain

$$
\left[\Xi^{\langle-\vec{l}\rangle}, \Xi^{\langle-n\rangle}\right] \equiv 0, \quad \bar{l}=1, \ldots, n-1 .
$$


Substituting (55) into (61) and taking into account the product derivative formula $\left[\Xi^{\langle-l\rangle}, a_{n} \Xi\right]=\left\langle\mathrm{d} a_{n}, \Xi^{\langle-l\rangle}\right\rangle \Xi+$ $a_{n}\left[\Xi^{\langle-l\rangle}, \Xi\right]$ and (53), we can rewrite (61) as

$$
\left\langle\mathrm{d} a_{n}, \Xi\langle-\vec{l}\rangle\right\rangle \Xi \equiv 0, \quad \bar{l}=1, \ldots, n-1
$$

This is possible iff $\left\langle\mathrm{d} a_{n}, \Xi\langle-\bar{l}\rangle\right\rangle \equiv 0$ for $\bar{l}=1, \ldots, n-1$. Shifting the last formula forward and taking again $l=$ $\bar{l}-1$, we get (59).

\section{EXAMPLES}

Example 13. Consider the system

$$
\begin{aligned}
& x_{1}^{\langle 1\rangle}=\frac{x_{1}^{2} u+x_{2}}{x_{1}}, \quad x_{2}^{\langle 1\rangle}=\frac{x_{3}\left(x_{1}^{2} u+x_{2}\right)}{x_{2}}, \\
& x_{3}^{\langle 1\rangle}=\frac{x_{1} x_{3} u^{2}}{x_{2}}, \quad y=x_{1},
\end{aligned}
$$

where $x_{i}>0, i=1,2,3, u>0$. The spaces $\bar{X}$ and $U$ are defined by the above inequalities. Observe that

$$
\begin{array}{r}
\operatorname{rank}_{\mathscr{K}} \frac{\partial \bar{\Phi}}{\partial x} \\
=\operatorname{rank}_{\mathscr{K}}\left(\begin{array}{ccc}
\frac{x_{1}^{2} u-x_{2}}{x_{1}^{2}} & \frac{1}{x_{1}} & 0 \\
\frac{2 x_{1} x_{3} u}{x_{2}} & -\frac{x_{1}^{2} x_{3} u}{x_{2}^{2}} & \frac{x_{1}^{2} u+x_{2}}{x_{2}} \\
\frac{x_{3} u^{2}}{x_{2}} & -\frac{x_{1} x_{3} u^{2}}{x_{2}^{2}} & \frac{x_{1} u^{2}}{x_{2}}
\end{array}\right)=2,
\end{array}
$$

meaning that the system is non-reversible. Take $z=$ $h(x)=x_{1}$, then

$$
\begin{aligned}
& x_{1}^{\langle-1\rangle}=z^{\langle-1\rangle}, x_{2}^{\langle-1\rangle}=x_{1} z^{\langle-1\rangle}-\left(z^{\langle-1\rangle}\right)^{2} \sqrt{\frac{x_{1} x_{3}}{x_{2}}}, \\
& x_{3}^{\langle-1\rangle}=x_{2}-z^{\langle-1\rangle} \sqrt{\frac{x_{2} x_{3}}{x_{1}}}, \quad u^{\langle-1\rangle}=\sqrt{\frac{x_{1} x_{3}}{x_{2}}} .
\end{aligned}
$$

Find the vector field $\Xi$, defined by (20). Compute, according to (18),

$$
\begin{gathered}
\omega_{0}=\mathrm{d} x_{1}, \quad \omega_{1}=\frac{x_{1}^{2} u-x_{2}}{x_{1}^{2}} \mathrm{~d} x_{1}+\frac{1}{x_{1}} \mathrm{~d} x_{2}, \\
\omega_{2}=\frac{x_{1}^{2}\left(x_{2} u u^{\langle 1\rangle}+x_{3}\right)-x_{2}^{2} u^{\langle 1\rangle}}{x_{1}^{2} x_{2}} \mathrm{~d} x_{1} \\
+\frac{x_{2}^{2} u^{\langle 1\rangle}-x_{1}^{2} x_{3}}{x_{1} x_{2}^{2}} \mathrm{~d} x_{2}+\frac{x_{1}}{x_{2}} \mathrm{~d} x_{3},
\end{gathered}
$$

and solve (20) to obtain

$$
\Xi=\frac{x_{2}}{x_{1}} \frac{\partial}{\partial x_{3}} .
$$

Compute next the projections of the backward shifts of $\Xi$ up to the order $n=3$. According to (8), (9) and (10) we get

$$
\begin{aligned}
& \Xi^{\langle-1\rangle \pi}=x_{1} \frac{\partial}{\partial x_{2}}+\frac{x_{1} x_{3}}{x_{2}} \frac{\partial}{\partial x_{3}}, \\
& \Xi^{\langle-2\rangle \pi}=\frac{\partial}{\partial x_{1}}+\frac{x_{2}}{x_{1}} \frac{\partial}{\partial x_{2}}, \\
& \Xi^{\langle-3\rangle \pi}=\sqrt{\frac{x_{1} x_{3}}{x_{2}}} \frac{\partial}{\partial x_{1}}+\sqrt{\frac{x_{2} x_{3}}{x_{1}}} \frac{\partial}{\partial x_{2}} .
\end{aligned}
$$

Check the conditions of Theorem 6. Since the vector fields $\Xi, \Xi^{\langle-1\rangle \pi}$ and $\Xi^{\langle-2\rangle \pi}$ commute, the condition (i) of Theorem 6 is satisfied. Observe that the coefficients of the above vector fields depend only on the variable $x$, thus also (ii) holds. From (63)

$$
\Xi^{\langle-3\rangle \pi}=\sqrt{\frac{x_{1} x_{3}}{x_{2}}} \Xi^{\langle-2\rangle \pi},
$$

and from (62) one can see that $\sqrt{x_{1} x_{3} / x_{2}}=u^{\langle-1\rangle}$. Consequently, also (iii) is valid and the system is transformable into the observer form (17). Construct

$$
M=\left[\Xi^{\langle-2\rangle \pi} \Xi^{\langle-1\rangle \pi} \Xi\right]=\left(\begin{array}{ccc}
1 & 0 & 0 \\
\frac{x_{2}}{x_{1}} & x_{1} & 0 \\
0 & \frac{x_{1} x_{3}}{x_{2}} & \frac{x_{2}}{x_{1}}
\end{array}\right)
$$

and find its inverse

$$
M^{-1}=\left[\begin{array}{l}
\mathrm{d} X_{1} \\
\mathrm{~d} X_{2} \\
\mathrm{~d} X_{3}
\end{array}\right]=\left(\begin{array}{ccc}
1 & 0 & 0 \\
-\frac{x_{2}}{x_{1}^{2}} & \frac{1}{x_{1}} & 0 \\
\frac{x_{3}}{x_{2}} & -\frac{x_{1} x_{3}}{x_{2}^{2}} & \frac{x_{1}}{x_{2}}
\end{array}\right),
$$

leading to the state transformation $X_{1}=x_{1}, X_{2}=x_{2} / x_{1}$, $X_{3}=x_{1} x_{3} / x_{2}, y=X_{1}$. The new state equations in the form (17) are

$X_{1}^{\langle 1\rangle}=X_{2}+u y, \quad X_{2}^{\langle 1\rangle}=X_{3}, \quad X_{3}^{\langle 1\rangle}=u^{2}, \quad y=X_{1}$.

Instead of checking linear independence as in (64), one may alternatively use the formula (50) and check whether the elements of the resulting matrix depend only on $y^{\langle-1\rangle}$ and $u^{\langle-1\rangle}$. Write $\Xi^{\langle-n\rangle \pi}$ in the form of a column vector

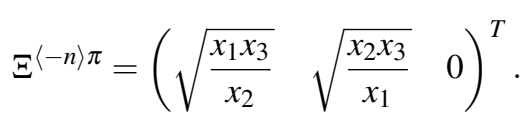


Then

$$
M^{-1} \Xi^{\langle-n\rangle \pi}=\left(\sqrt{\frac{x_{1} x_{3}}{x_{2}}} \quad 0 \quad 0\right)^{T}=\left(\begin{array}{llll}
u^{\langle-1\rangle} & 0 & 0
\end{array}\right)^{T} .
$$

The elements of the obtained matrix depend only on $u^{\langle-1\rangle}$ and therefore the condition (iii) of Theorem 6 really holds.

Example 14. Consider the system

$$
x_{1}^{\langle 1\rangle}=x_{2}, \quad x_{2}^{\langle 1\rangle}=\left(1+x_{3}\right) u, \quad x_{3}^{\langle 1\rangle}=u, \quad y=x_{1},
$$

where $\bar{X}=\mathbb{R}^{3} \backslash[(\varnothing),(\varnothing),(0)], U=\mathbb{R} \backslash(0)$. Observe that the system is non-reversible since

$$
\operatorname{rank}_{\mathscr{K}}\left(\frac{\partial \bar{\Phi}}{\partial x}\right)=\operatorname{rank}_{\mathscr{K}}\left(\begin{array}{ccc}
0 & 1 & 0 \\
0 & 0 & u \\
0 & 0 & 0
\end{array}\right)=2
$$

The choice $z=h(x)=x_{1}$ results in $x_{1}^{\langle-1\rangle}=z^{\langle-1\rangle}, x_{2}^{\langle-1\rangle}=$ $x_{1}, x_{3}^{\langle-1\rangle}=\left(x_{2}-x_{3}\right) / x_{3}, u^{\langle-1\rangle}=x_{3}$. Compute, according to (18), $\omega_{0}=\mathrm{d} x_{1}, \omega_{1}=u \mathrm{~d} x_{3}, \omega_{2}=u \mathrm{~d} x_{3}$. Find the vector field $\Xi$ from (20):

$$
\Xi=\frac{1}{u} \frac{\partial}{\partial x_{3}} .
$$

The condition (ii) of Theorem 6 is not satisfied since the coefficient of $\Xi$ depends on $u$. Therefore, the system can not be transformed into the observer form. We will demonstrate what will happen if we try to find the state transformation and transformed equations. Of course, we can still compute $\Xi^{\langle-1\rangle \pi}=\partial / \partial x_{2}, \Xi^{\langle-2\rangle \pi}=\partial / \partial x_{1}$. The vector fields $\Xi, \Xi^{\langle-1\rangle \pi}$ and $\Xi^{\langle-2\rangle \pi}$ commute, but (25) does no longer give us the state transformation (in $n$ dimensional space):

$$
X_{1}=x_{1} u, \quad X_{2}=x_{2}, \quad X_{3}=x_{3} .
$$

If we defined, using (65), the coordinate transformation in $(n+m)$-dimensional space with coordinates $\{x, u\}$ and take $\{X, u\}$ as the new coordinates, the transformed equations would take the form

$$
X_{1}^{\langle 1\rangle}=X_{2}, \quad X_{2}^{\langle 1\rangle}=X_{3}+u, \quad X_{3}^{\langle 1\rangle}=u u^{\langle 1\rangle} .
$$

\section{COMPARISON WITH EARLIER CONDITIONS}

In this section we compare the conditions of Corollary 12 with those from [6] under two restrictive assumptions made in [6] and recalled below. Though, by Assumption 3 , the dimension of the observable space $\mathscr{O}$ is generically equal to $n$, there may exist the so-called singular (non-regular) points where the dimension drops, i.e. is less than $n$. In such points the results of [6] cannot be used. Denote $\mathbf{u}:=\left\{u, \ldots, u^{\langle n-1\rangle}\right\}$ and $\mathbf{u}_{l-1}:=\left\{u^{\langle i\rangle}, i=\right.$ $0, \ldots, l-1\}$.
Assumption 15. The point $x=0, \mathbf{u}=0$ is the regular point of the observable space.

Moreover, since the results of [6] hold locally around $x=0, \mathbf{u}=0$, for comparison, we have to assume that the point $(0,0) \in \bar{X} \times U$.

Introduce the notation $\bar{\Phi}_{0}(x):=\bar{\Phi}(x, 0)$. For $i \geq 0$, $\bar{\Phi}_{0}^{i+1}:=\bar{\Phi}_{0}^{i}\left(\bar{\Phi}_{0}(x)\right)$, where $\bar{\Phi}_{0}^{0}=$ Id. Under Assumption 15 , the generic observability condition (19) implies that the so-called strong observability condition

$$
\operatorname{dim}_{\mathscr{R}}\left(\operatorname{span} \mathscr{R}\left\{\mathrm{d} h, \mathrm{~d}\left(h \circ \bar{\Phi}_{0}\right), \ldots, \mathrm{d}\left(h \circ \bar{\Phi}_{0}^{n-1}\right)\right\}\right)=n,
$$

made in [6], holds in the neighbourhood of $x=0$, because $\left.\omega_{l}\left(x, \mathbf{u}_{l-1}\right)\right|_{\mathbf{u}_{l-1}=0}=\mathrm{d}\left(h \circ \bar{\Phi}_{0}^{l}(x)\right)$.

Assumption 16. In the neighbourhood of the point $x=0$

$$
\operatorname{rank}_{\mathscr{R}}\left(\frac{\partial \bar{\Phi}_{0}(x)}{\partial x}\right)=n
$$

Under Assumption 16 exists $\bar{\Phi}_{0}^{-1}$, which in our earlier notation is $\Lambda(x, 0)$. To present Theorem 1 from [6], recall the vector field $r_{1} \in \operatorname{span}_{\mathscr{R}}\{\partial / \partial x\}$, defined by

$$
\left\langle\mathrm{d}\left(h \circ \bar{\Phi}_{0}^{l}\right), r_{1}\right\rangle \equiv \delta_{l, n-1}, \quad l=0, \ldots, n-1,
$$

and the operator $\operatorname{Ad}_{\bar{\Phi}_{0}}$, applicable to the vector fields $\widehat{\Xi}=\sum_{i=1}^{n} \widehat{a}_{i}(x) \partial / \partial x_{i} \in \operatorname{span}_{\mathscr{K}}\{\partial / \partial x\}$ and defined by

$$
\begin{aligned}
& \operatorname{Ad}_{\bar{\Phi}_{0}} \widehat{\Xi} \\
& =\left.\sum_{i, j=1}^{n} \frac{\partial \bar{\Phi}_{0 i}\left(x^{\langle-1\rangle}\right)}{\partial x_{j}^{\langle-1\rangle}} \widehat{a}_{j}\left(x^{\langle-1\rangle}\right)\right|_{x^{\langle-1\rangle}=\bar{\Phi}_{0}(x)} \frac{\partial}{\partial x_{i}} .
\end{aligned}
$$

Moreover, recall that

$$
r_{i}:=\operatorname{Ad}_{\bar{\Phi}_{0}}^{i-1} r_{1}, \quad i=2, \ldots, n,
$$

and the vector field $G^{0}\left(x, u^{\langle-1\rangle}\right):=$ $\left.\left(\partial \bar{\Phi}\left(x^{\langle-1\rangle}, u^{\langle-1\rangle}\right) / \partial u^{\langle-1\rangle}\right)\right|_{x^{\langle-1\rangle}=\Lambda\left(x, u^{\langle-1\rangle}\right)}$ and

$$
\begin{aligned}
G^{0}\left(x, u^{\langle-1\rangle}\right) & :=\left.\frac{\partial \bar{\Phi}\left(x^{\langle-1\rangle}, u^{\langle-1\rangle}\right)}{\partial u^{\langle-1\rangle}}\right|_{x^{\langle-1\rangle}=\Lambda\left(x, u^{\langle-1\rangle}\right)} \\
& =G_{1}^{0}(x)+\sum_{k \geq 1} G_{k+1}^{0}(x) \frac{\left(u^{\langle-1\rangle}\right)^{k}}{k !}
\end{aligned}
$$

where

$G_{1}^{0}=G_{0}(x, 0)$,

$G_{k}^{0}(x):=\left.\frac{\partial^{k-1}}{\partial\left(u^{\langle-1\rangle}\right)^{k-1}} G^{0}\left(x, u^{\langle-1\rangle}\right)\right|_{u^{\langle-1\rangle}=0}, \quad k>1$. 
Lemma 17. $G^{0}=(\partial / \partial u)^{\langle-1\rangle \pi}$.

Proof. The proof follows directly from Lemma 11.

Theorem 18. [6] Under Assumptions 15 and 16, the system (16) is locally transformable into the observer form (17) via a coordinate transformation $X=\Psi(x)$ if and only if the following conditions are satisfied:

(A) $\left[r_{1}, r_{i+1}\right] \equiv 0, i=1, \ldots, n-1$,

(B) $\left[G_{k}^{0}, r_{i+1}\right] \equiv 0, i=1, \ldots, n-1, k \geq 0$.

We will show that the conditions of Corollary 12 , restricted to a neighbourhood of the point $x=0, \mathbf{u}=0$, under Assumptions 15 and 16, yield to those of Theorem 18. First, we prove some lemmas.

Lemma 19. Under Assumption 15, if the condition (ii*) of Corollary 12 is satisfied, then $\Xi=r_{1}$.

Proof. We will show that under the assumptions of the lemma the equations (20) that define the vector field $\Xi$ reduce to

$$
\left\langle\mathrm{d}\left(h \cdot \bar{\Phi}_{0}^{l}\right), \Xi\right\rangle \equiv \delta_{l, n-1}, \quad l=0, \ldots, n-1,
$$

which are the same that define $r_{1}$ in (67). By (ii*) the coefficients of the 1-forms $\omega_{l}, l=0, \ldots, n-1$, (and therefore also $\Xi$ ) in (20) depend only on the variable $x$. Observe next that under Assumption 15 one may take $\mathbf{u}=0$ in the coefficients of $\omega_{l}$ because this does not affect the result. This change, due to (66), gives (71).

Proposition 20. In the neighbourhood of $x=0$, under the mild assumption that $(0,0) \in \bar{X} \times U$

$$
\frac{\partial \bar{\Phi}_{0}(x)}{\partial x}=\left.\frac{\partial \bar{\Phi}(x, u)}{\partial x}\right|_{u=0} .
$$

Proof. Since $\bar{\Phi}$ is analytic, one can expand the function into the Taylor series around $u=0$ under the mild assumption that $0 \in U$ :

$$
\bar{\Phi}(x, u)=\bar{\Phi}_{0}(x)+\left.\sum_{k>0} \frac{\partial^{k} \bar{\Phi}(x, u)}{\partial u^{k}}\right|_{u=0} \frac{u^{k}}{k !} .
$$

Then

$$
\frac{\partial \bar{\Phi}(x, u)}{\partial x}=\frac{\bar{\Phi}_{0}(x)}{\partial x}+\sum_{k>0} \frac{\partial}{\partial x}\left(\left.\frac{\partial^{k} \bar{\Phi}(x, u)}{\partial u^{k}}\right|_{u=0}\right) \frac{u^{k}}{k !}
$$

and (72) is easily seen.

Lemma 21. Under Assumption 16, if the condition ( $\left.i i^{*}\right)$ of Corollary 12 is satisfied, then $r_{l+1}=\Xi^{\langle-l\rangle}, l=$ $1, \ldots, n-1$.

Proof. We begin by proving that

$$
\Xi^{\langle-l\rangle}=\operatorname{Ad}_{\Phi_{0}}^{l} \Xi, \quad l=0, \ldots, n-1 .
$$

By (20), $\Xi \in \operatorname{span}_{\mathscr{K}}\{\partial / \partial x\}$ and due to Lemma 10, this is also true for all its backward shifts. Additionally, according to (ii*) the coefficients of the vector field depend only on $x$, i.e. $\Xi^{\langle-l\rangle}=\sum_{l=1}^{n} \xi_{i, l}(x) \partial / \partial x_{i}, l=0, \ldots, n-1$. If in (52) one takes $\widehat{\Xi}=\Xi^{\langle-l+1\rangle}$, we get

$$
\Xi^{\langle-l\rangle}=\sum_{i=1}^{n}\left\langle\mathrm{~d} \bar{\Phi}_{i}, \Xi^{\langle-l+1\rangle}\right\rangle^{\langle-1\rangle} \frac{\partial}{\partial x_{i}} .
$$

Rewrite (74), using the definition of scalar product, as

$$
\begin{aligned}
\Xi^{\langle-l\rangle}= & \sum_{i, j=1}^{n} \frac{\partial \bar{\Phi}_{i}\left(x^{\langle-1\rangle}, u^{\langle-1\rangle}\right)}{\partial x_{j}^{\langle-1\rangle}} \\
& \times\left.\xi_{j, l-1}\left(x^{\langle-1\rangle}\right)\right|_{x^{\langle-1\rangle}=\Lambda\left(x, u^{\langle-1\rangle}\right)} \frac{\partial}{\partial x_{i}} .
\end{aligned}
$$

On the right hand side of (75) the coefficients of the vector field do not depend on $u^{\langle-1\rangle}$, and so nothing will change if we take $u^{\langle-1\rangle}=0$. Moreover, by Proposition 20 replace in (75) $\bar{\Phi}$ by $\bar{\Phi}_{0}$ and by Assumption 16, replace $\Lambda(x, 0)$ by $\bar{\Phi}_{0}^{-1}(x)$ to yield

$$
\begin{aligned}
\Xi^{\langle-l\rangle}= & \sum_{i, j=1}^{n} \frac{\partial \bar{\Phi}_{0 i}\left(x^{\langle-1\rangle}\right)}{\partial x_{j}^{\langle-1\rangle}} \\
& \times\left.\xi_{j, l-1}\left(x^{\langle-1\rangle}\right)\right|_{x^{\langle-1\rangle}=\bar{\Phi}_{0}^{-1}(x)} \frac{\partial}{\partial x_{i}} .
\end{aligned}
$$

Comparing (76) with (68), one can conclude that

$$
\Xi^{\langle-l\rangle}=\operatorname{Ad}_{\bar{\Phi}_{0}} \Xi^{\langle-l+1\rangle} .
$$

From (77) the validity of (73) follows. Finally, $r_{l+1}=$ $\Xi^{\langle-l\rangle}$ results from (69).

The above lemmas allow to show that the results of [6], recalled in this paper as Theorem 18, follow from our results for reversible systems, as given in Corollary 12 .

The condition (A) of Theorem 18 follows from the condition (i*) of Corollary 12, due to Lemmas 19 and 21 if (ii*) holds. Next we will prove that the condition (B) follows from (ii*). The condition (B) is, due to (70), equivalent to

$$
\left[G^{0}, r_{l+1}\right] \equiv 0, \quad l=1, \ldots, n-1 .
$$

Rewrite (78), due to Lemmas 17, 19 and 21, in the form

$$
\left[\left(\frac{\partial}{\partial u}\right)^{\langle-1\rangle \pi}, \Xi^{\langle-l\rangle}\right] \equiv 0, \quad l=1, \ldots, n-1 .
$$

That is, we have to show that from the validity of (ii*) follows (79). Obviously (ii*) implies that

$$
\left[\frac{\partial}{\partial u}, \Xi\langle-\bar{l}\rangle\right] \equiv 0, \quad \bar{l}=0, \ldots, n-2 .
$$


Shifting (80) backward and denoting $l:=\bar{l}+1$, we obtain an equivalent formula

$$
\left[\left(\frac{\partial}{\partial u}\right)^{\langle-1\rangle}, \Xi^{\langle-l\rangle}\right] \equiv 0, \quad l=1, \ldots, n-1 .
$$

According to Lemma 11 one can rewrite (81), for $l=$ $1, \ldots, n-1$, as

$$
\left[\left(\frac{\partial}{\partial u}\right)^{\langle-1\rangle \pi}, \Xi^{\langle-l\rangle}\right]+\left[\frac{\partial}{\partial u^{\langle-1\rangle}}, \Xi^{\langle-l\rangle}\right] \equiv 0,
$$

where

$$
\left(\frac{\partial}{\partial u}\right)^{\langle-1\rangle \pi}=\left.\sum_{i=1}^{n} \frac{\partial \bar{\Phi}_{i}\left(x^{\langle-1\rangle}, u^{\langle-1\rangle}\right)}{\partial u^{\langle-1\rangle}}\right|_{x^{\langle-1\rangle}=\Lambda\left(x, u^{\langle-1\rangle}\right)} \frac{\partial}{\partial x_{i}} .
$$

The second term on the left hand side of (82) equals identically zero due to (ii*), therefore (79) holds.

\section{CONCLUSIONS}

The paper has studied the problem of transforming the discrete-time state equations into the observer form. The necessary and sufficient conditions for the existence of the state transformation are formulated in terms of the backward shifts of the vector fields, defined by the system dynamics, for the case when the state equations are not necessarily reversible (with respect to the state variable). The method to find the required state transformation is also provided. The obtained conditions are then compared with those obtained earlier under the more restrictive assumption of reversibility of the state dynamics [6].

For simplicity of presentation, this paper has addressed only the single-input single-output case. Extension to the multi-input multi-output case does not include difficulties but is, of course, technically much more involved. However, the interesting research perspectives include extension of the results to find the conditions under which the state equations can be transformed, using the parametrized state transformation, into the extended observer form. Such a form consists of linear equations up to nonlinear terms that depend, besides inputs and outputs like in the case of classical observer form, also on their backward shifts up to certain order.

\section{ACKNOWLEDGEMENTS}

The work of Ü. Kotta was partially supported by the Estonian Centre of Excellence in IT funded by the European Regional Development Fund. The publication costs of this article were covered by the Estonian Academy of Sciences.

\section{REFERENCES}

1. Kaparin, V. and Kotta, Ü. Transformation of nonlinear discrete-time system into the extended observer form. Int. J. Control, 2018, 91(4), 848-858.

2. Lee, H.-G., Arapostathis, A. and Marcus, S. I. Necessary and sufficient conditions for state equivalence to a nonlinear discrete-time observer canonical form. IEEE Trans. Autom. Control, 2008, 53(11), 2701-2707.

3. Lee, H.-G. and Hong, J.-M. Algebraic conditions for state equivalence to a discrete-time nonlinear observer canonical form. Syst. Control Lett., 2011, 60(9), 756762.

4. Mullari, T. and Kotta, Ü. Transformation the nonlinear system into the observer form: simplifications and extension. Eur. J. Control, 2009, 15(2), 177-183.

5. Lee, H. G. Verifiable conditions for discrete-time multi output observer error linearizability. IEEE Trans. Autom. Control, 2019, 64(4), 1632-1639.

6. Califano, C., Monaco, S. and Normand-Cyrot, D. Canonical observer forms for multi-output systems up to coordinate and output transformations in discrete time. $\mathrm{Au}$ tomatica, 2009, 45(11), 2483-2490.

7. Califano, C., Monaco, S. and Normand-Cyrot, D. On the observer design in discrete-time. Syst. Control Lett., 2003, 49(4), 255-265.

8. Mullari, T., Kotta, Ü., Bartosiewicz, Z., Pawluszewicz, E. and Moog, C. H. Forward and backward shifts of vector fields: towards the dual algebraic framework. IEEE Trans. Autom. Control, 2017, 62(6), 3029-3033.

9. Aranda-Bricaire, E., Kotta, Ü. and Moog, C. H. Linearization of discrete-time systems. SIAM J. Control. Optim., 1996, 34(6), 1999-2023.

10. Mullari, T. and Kotta, Ü. Linearization of discrete-time control systems by state transformation. Proc. Est. Acad. Sci., 2021, 70(1), 62-79.

11. Kotta, Ü., Schlacher, K. and Tõnso, M. Relaxing realizability conditions for discrete-time nonlinear systems. $A u$ tomatica, 2015, 58, 67-71. 


\title{
Mittelineaarsete diskreetsete olekuvõrrandite teisendamine vaatlejakujule: üldistus mittepööratavale juhule
}

\author{
Tanel Mullari ja Ülle Kotta
}

On käsitletud diskreetajaga olekuvõrrandite teisendamist vaatlejakujule. Esmalt on tõestatud vaatlejakuju olemasolu tarvilikud ja piisavad tingimused süsteemi dünaamika defineeritud vektorväljade tagasinihete kaudu. Antud tingimuste eelis olemasolevate ees seisneb selles, et need ei eelda süsteemi pööratavust olekumuutujate suhtes. Seejärel on antud meetod olekuteisenduse leidmiseks, mis võimaldab süsteemi vaatlejakujule viia. Saadud tingimusi on võrreldud varasematega, mis on tuletatud süsteemi pööratavust eeldades. Artiklis esitatud teooria on illustreeritud kahe näitega. 